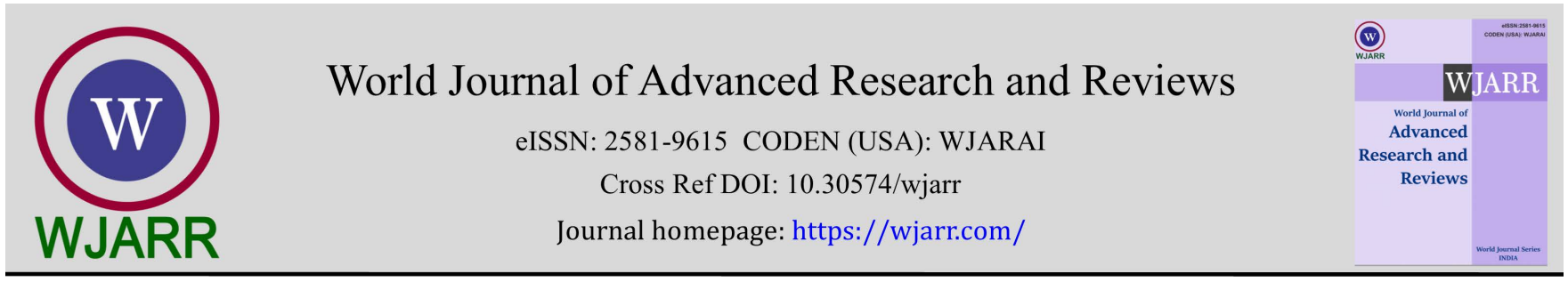

(RESEARCH ARTiClE)

\title{
Investigating the status of knowledge dissemination and transfer among the managers of the Ministry of Health and Medical Education in the process of coping and disease management especially COVID 19
}

Hossein Kazemeini 1, Ahad Banar 2, *, Morteza Gholnezhad ${ }^{3}$, Jamshid Mehrabi 4, Maedeh Mahmoudi Pour 5 , Zohreh Ghasemian ${ }^{6}$ and Maliheh Molaie Maran ${ }^{7}$

\author{
${ }^{1}$ Social Medicine Specialist, Faculty Member and Assistant Professor, Ministry of Health and Medical Education, Tehran, \\ Iran. \\ 2 Ph.D. in Public Policy Management, Deputy of Public Health ‘Ministry of Health and Medical Education and Department \\ of Public Management, Central Tehran Branch, Islamic Azad University, Tehran, Iran. \\ ${ }^{3}$ Ph. D in public policy making, university of applied science and technology \\ ${ }^{4}$ Faculty of management, university of ghaemshahr, Mazandaran, Iran. \\ ${ }^{5}$ Department of PHC, Torbat-e Heydariyyeh Medical University, Iran. \\ ${ }^{6}$ Management and health economy department, faculty of health, Tehran Medical University Tehran, Iran. \\ 7 Department of PHC, Jiroft Medical University, Iran.
}

World Journal of Advanced Research and Reviews, 2022, 13(02), 292-298

Publication history: Received on 30 December 2021; revised on 06 February 2022; accepted on 08 February 2022

Article DOI: https://doi.org/10.30574/wjarr.2022.13.2.0125

\begin{abstract}
Background and Aim: The importance of developing a strategy at the organization level in the face of complex environments has been discussed in various researches but the need to pay attention to them is so great that it will be very effective in achieving organizational excellence. The purpose of this study is to select the top strategy in the Ministry of Health based on the model of the strategic planning process based on prescriptive attitudes.
\end{abstract}

Methods :The present study is an applied study. The study conducted in 2017-2018 in the operational planning headquarters of the Ministry of Health with a statistical population of 33 planning managers. In terms of collecting data, is descriptive and the field study. Research method is library based, Scrolling data and interview studies.

Results: Among the four strategies outlined: increased access to services, trained staffing, improved awareness and increased community health literacy, and appropriate infrastructure for equally benefiting from services, the strategy for increasing access to services, with 136 points in the Strategic planning Stakeholder Model Matrix and 4.6 in the Quantitative Strategy Planning Matrix (QSPM), designated as the top strategy in the Ministry of Health and medical education.

Conclusions :The results of this research can improve performance in today's complex organization environment. The selection of the Ministry of Health's best strategy is one of the findings of this study that helps university directors and affiliated organizations to formulate their own plans based on this strategy.

Keywords: Knowledge management; Knowledge dissemination; Knowledge transfer; Ministry of Health and Medical Education

\footnotetext{
* Corresponding author: Banar Ahad; E mail: ahadbanar@gmail.com

Public Policy Management, Deputy of Public Health، Ministry of Health and Medical Education and Department of Public Management, Central Tehran Branch, Islamic Azad University, Tehran, Iran 


\section{Introduction}

The ability of managers is attributed to their organizational skills, knowledge and experience, which is used in complex and difficult matters [1]. This creates a competitive advantage for the organization [2]. On the other hand, managers today are aware that knowledge resources are increasingly essential in the development of their organizations [3]. But the pervasive inability of management to apply knowledge as a resource has led to a number of new initiatives. Thus, most companies now have an intellectual capital manager, a knowledge manager, or an intellectual property or knowledge asset manager [4].

In this regard, providing health services to patients is a complex endeavor, which is increasingly dependent on knowledge and information, and just as people, materials and financial resources need management, knowledge and information are also a source that effective management should be applied to them by health care managers [5] Health care organizations need to collect and analyze data and turn it into information and knowledge for useful decision making [6]. Due to the ignorance of the results of applying the principles of knowledge management in the existing processes in the organization [7], the need for change in the principles and methods of management is obvious [8]. Because knowledge management is extremely important, in our country, like other countries that want development and progress, solid steps must be taken in the field of knowledge management and neglecting it will be synonymous with backwardness and underdevelopment [ 7].

Therefore, the law of the Fourth Economic, Social and Cultural Development Plan of the Islamic Republic of Iran also emphasizes the modernization of management methods [9], one of which is knowledge management. According to Evachmi Leka (2004), one of the characteristics of knowledge management is the dissemination of knowledge, including the division and distribution of knowledge. Knowledge dissemination does not always take place in the form of the formal hierarchical structure of the organization, but also knowledge transfer takes place in the network of informal structure (6). However, in developed countries, one of the obvious examples is the dissemination of knowledge of internships. In these courses, skills are transferred to the staff in the form of special training from prof essors [10].

In this regard, researches has proven the relationship between knowledge management and innovation in the organization and knowledge management and high organizational performance [11]. In view of what has been said, also due to the low level of familiarity and application of knowledge management principles in health care organizations [7], especially in crisis situations and in the face of outbreaks such as Covid disease 19 and little research in this area. [6] and the vitality of knowledge transfer in the organization [12], especially in the face of disease and management of diseases such as Covid 19, the most important issue that has occupied the minds of researchers is the status of knowledge dissemination and transfer among the staff managers of the Ministry of Health and Medical Education at the time of exposure and management of the epidemic of this disease (Covid 19)?

\section{Methods}

The research method was descriptive with a qualitative and quantitative approach that in terms of purpose, also had a practical and developmental aspect. The statistical population of the study is the staff managers of the Ministry of Health and Medical Education who have been directly related to the management of Covid 19 disease epidemic. In the qualitative part of the research, using open interview, the components of knowledge dissemination and transfer in the field of exposure and management of Covid disease were determined and approved based on the views of experts in the field of knowledge management. Then a questionnaire of 8 questions was designed based on it. In order to determine the validity of the questionnaire, first the questionnaire was distributed among $25 \%$ of the statistical population (staff managers) to eliminate possible problems and finally and after this stage, the validity of the questionnaire was finally approved by the consulting professors.

To calculate the reliability coefficient, Cronbach's alpha measurement method was used by SPSS software. The obtained alpha value is equal to $79 \%$, which is a statistically desirable value and indicates the reliability and validity of the questionnaire. As pointed out by the statistical population considered in the study of the quantitative section, staff managers are concerned with the issue of epidemiological management of Covid disease. After determining the statistical population, it is time to determine the statistical sample or the number of respondents and select them. For this purpose, the method proposed by Parker and Henry (1990) is used to determine the minimum sample. This method is available and can be used according to the table provided by the two. In this method, and based on the table provided, we have: If the total population is less than 200. Namely: $\mathrm{N}<200$

the $95 \%$ confidence interval means: $\mathrm{e}=95 \%$ 
considering the margin of error of $3 \%$, that is: $r=3 \%$

In this case, the minimum number of samples is equal to the whole population, ie $\mathrm{N}^{*}=\mathrm{N}=80$

In fact, this means that in the model proposed by Parker and Henry, If the total number of population sampled is less than 200, the entire community should be sampled and surveyed. As a result, in order to be able to study the community by researchers, sampling was not done and by referring in person and sending electronic questionnaires anonymously among staff managers related to the process of exposure and management of Covid 19 disease, encouragement and frequent follow-up were performed to complete the questionnaire.

Data collection tools were an open-ended questionnaire in the first part (qualitative) and a questionnaire based on the five Likert spectrum (in the quantitative part), which consists of two parts. Part 1: Demographic data (age, gender, service history, experience in managerial jobs, employment status and job of managers). Part 2: The questionnaire developed based on the first part of the research includes the component of dissemination and transfer of knowledge during the epidemic of Covid 19 disease among managers. In the present study, two components of knowledge dissemination and transfer were used to collect data. The questionnaire had 8 questions in the Likert scale (five options including very low, low, somewhat, high and very high) in which the component of knowledge dissemination was examined by 4 questions and knowledge transfer was examined by 4 similar questions.

In order to assess the validity of the questionnaire, the expert opinion method was used, so that the questionnaire was prepared for a number of professors who had a history of research and study in the field of research and they were asked to express their views on the content. The structure and method of writing the questionnaire. Then, by attending and discussing the questions of the questionnaire and after applying the suggestions and its approval by other members, the validity of the content and structure of the questionnaire was guaranteed. The reliability of the questionnaire was calculated using SPSS software and Cronbach's alpha was 0.79, which indicates the reliability of the questionnaire. Finally, descriptive statistics (mean, median and variance) were used to analyze the data.

\section{Results}

Demographic data include age, sex, service history, managerial service, employment status and level of education, which are summarized in the table below. It should be noted that service and managerial history are grouped in three groups of low, medium and high and age in four equal groups, which is presented in Table 1

Table 1 Demographic characteristics of managers related to Covid 19 disease management in the present study

\begin{tabular}{|c|c|c|c|}
\hline \multicolumn{2}{|c|}{ Frequency of demographic characteristics } & \multicolumn{2}{|c|}{ Abundance } \\
\hline & & \multirow{2}{*}{$\begin{array}{ll}\text { Number } & \\
& 18\end{array}$} & \multirow{2}{*}{$\begin{array}{l}\text { Rate } \\
\\
\end{array} 22.5$} \\
\hline Age(Year) & $25-35$ & & \\
\hline & $35-45$ & 42 & 52.5 \\
\hline & $45-55$ & 14 & 17.5 \\
\hline & Over 55 & 6 & 7.5 \\
\hline \multirow[t]{2}{*}{ Sex } & Female & 32 & 40 \\
\hline & Male & 48 & 60 \\
\hline \multirow[t]{3}{*}{ Years of service } & $5-15$ & 24 & 30 \\
\hline & $15-25$ & 32 & 40 \\
\hline & Over 25 & 24 & 30 \\
\hline \multirow{3}{*}{$\begin{array}{l}\text { Managerial background } \\
\text { (Year) }\end{array}$} & $1-10$ & 40 & 50 \\
\hline & $10-20$ & 20 & 25 \\
\hline & Over 20 & 20 & 25 \\
\hline Employment Status & Official hiring & 68 & 85 \\
\hline
\end{tabular}




\begin{tabular}{|l|l|c|c|}
\hline \multirow{3}{*}{ Education level } & Employment contract & 8 & 10 \\
\cline { 2 - 4 } & contractual employment & 4 & 5 \\
\cline { 2 - 4 } & Expert & 10 & 12.5 \\
\cline { 2 - 4 } & MA & 20 & 25 \\
\cline { 2 - 4 } & GP & 35 & 43.75 \\
\cline { 2 - 4 } & PHD & 15 & 18.75 \\
\hline
\end{tabular}

The method of answering the questions was as follows, the results of which are presented in Table 2:

Question 1: How much is the distribution and dissemination of knowledge in order to make it available to the public? This question emphasizes past experiences to improve quality and reduce errors. The study population showed the distribution and dissemination of knowledge at the time of exposure and management of Covid 19 disease as 57.5\% above average, $37.5 \%$ as average and $5 \%$ below average.

Question 2: To what extent does the organization encourage employees to disseminate and distribute knowledge in the organizational environment? This question emphasizes past experiences to improve quality and reduce errors. The study population evaluated the rate of knowledge dissemination and dissemination in the organizational environment and the time of exposure and management of Covid 19 disease as $67.5 \%$ above average, $20 \%$ as average and $12.5 \%$ below average.

Question 3: To what extent is knowledge disseminated in the organization through seminars, conferences and workshops (online and webinars)? This question emphasizes past experiences to improve quality and reduce errors. The study population showed the amount of knowledge distribution and dissemination in the form of seminars, conferences and workshops (webinar and online) at the time of exposure and management of Covid 19 disease as $72.5 \%$ above average, $17.5 \%$ as average and $10 \%$ below average.

Question 4: To what extent is knowledge disseminated in the organization through organizational rules, circulars and instructions? This question emphasizes past experiences to improve quality and reduce errors. The study population showed the rate of knowledge distribution through instructions and circulars at the time of exposure and management of Covid 19 disease as $60 \%$ above average, $35 \%$ as average and $5 \%$ below average.

Question 5: What is the rate of knowledge transfer in order to make it publicly available among the managers of the organization? This question emphasizes past experiences to improve quality and reduce errors. The study population showed the rate of knowledge transfer between managers of the organization during exposure and management of Covid 19 disease as $72.5 \%$ above average, $17.5 \%$ as average and $10 \%$ below average.

Question 6: To what extent does the organization encourage managers and employees to transfer knowledge to each other? This question emphasizes past experiences to improve quality and reduce errors. The study population rated the encouragement of managers and employees to transfer knowledge during exposure and management of Covid 19 disease as $60 \%$ above average, $35 \%$ as average and $5 \%$ below average.

Question 7: To what extent is knowledge transferred in the organization through face-to-face meetings and lectures (online and webinars)? This question emphasizes past experiences to improve quality and reduce errors. The study population showed the rate of knowledge transfer between managers through face-to-face meetings and lectures (online and webinar) at the time of exposure and management of Covid 19 disease $67.5 \%$ above average, $20 \%$ average and $12.5 \%$ below average .

Question 8: To what extent is knowledge transferred in the organization through organizational rules, regulations and instructions? This question emphasizes past experiences to improve quality and reduce errors. The study population showed the rate of knowledge transfer through rules, regulations and instructions at the time of exposure and management of Covid 19 disease as $57.5 \%$ above average, $37.5 \%$ as average and $5 \%$ below average. 
Table 2 Findings related to the knowledge dissemination component in the treatment and management of Covid disease 19

\begin{tabular}{|c|c|c|c|c|c|c|c|c|c|c|}
\hline option & \multicolumn{2}{|c|}{ Very Much } & \multicolumn{2}{c|}{ Much } & \multicolumn{2}{c|}{ Modium } & \multicolumn{2}{c|}{ Low } & \multicolumn{2}{c|}{ Very Low } \\
\hline Question & abundance & percent & abundance & percent & abundance & percent & abundance & percent & abundance & percent \\
\hline 1 & 6 & $\% 75$ & 40 & $\% 50$ & 30 & $\% 37.5$ & 2 & $\% 2.5$ & 2 & $\% 2.5$ \\
\hline 2 & 8 & $\% 10$ & 46 & $\% 57.5$ & 16 & $\% 20$ & 10 & $\% 12.5$ & 0 & $\% 0$ \\
\hline 3 & 16 & $\% 20$ & 42 & $\% 52.5$ & 14 & $\% 17.5$ & 4 & $\% 5$ & 4 & $\% 5$ \\
\hline 4 & 4 & $\% 5$ & 44 & $\% 55$ & 28 & $\% 35$ & 2 & $\% 2.5$ & 2 & $\% 2.5$ \\
\hline
\end{tabular}

Table 3 Findings related to the knowledge transfer component in the treatment and management of Covid disease 19

\begin{tabular}{|c|c|c|c|c|c|c|c|c|c|c|}
\hline \multirow{2}{*}{ option } & \multicolumn{2}{|c|}{ Very Much } & \multicolumn{2}{c|}{ Much } & \multicolumn{2}{c|}{ Modium } & \multicolumn{2}{c|}{ Low } & \multicolumn{2}{c|}{ Very Low } \\
\hline Question & abundance & percent & abundance & percent & abundance & percent & abundance & percent & abundance & percent \\
\hline 5 & 16 & $\% 20$ & 42 & $\% 52.5$ & 14 & $\% 17.5$ & 4 & $\% 5$ & 4 & $\% 5$ \\
\hline 6 & 4 & $\% 5$ & 44 & $\% 55$ & 28 & $\% 35$ & 2 & $\% 2.5$ & 2 & $\% 2.5$ \\
\hline 7 & 8 & $\% 10$ & 46 & $\% 57.5$ & 16 & $\% 20$ & 10 & $\% 12.5$ & 0 & $\% 0$ \\
\hline 8 & 6 & $\% 7.5$ & 40 & $\% 50$ & 30 & $\% 37.5$ & 2 & $\% 2.5$ & 2 & $\% 2.5$ \\
\hline
\end{tabular}

The average scores of knowledge dissemination and transfer components, along with its median and variance are presented in the table below

Table 4 Statistical indicators related to the components of dissemination and transfer of knowledge management in the face and management of Covid19 disease

\begin{tabular}{|l|c|c|c|c|c|c|}
\hline \multirow{2}{*}{ Variable } & \multicolumn{3}{|c|}{ Cumulative } & \multicolumn{3}{c|}{ Mean } \\
\cline { 2 - 7 } & Mean & Medium & Variance & Mean & Medium & Variance \\
\hline Dissemination of knowledge & 14.75 & 14 & 7.73 & 3.64 & 3.5 & 48 \\
\hline Transfer of knowledge & 14.75 & 14 & 7.73 & 3.64 & 3.5 & 48. \\
\hline
\end{tabular}

\section{Discussion}

According to the findings of the analysis of research data, it was found that the status of knowledge management during the process of exposure and management of Covid 19 disease in the headquarters of the Ministry of Health and Medical Education is a favorable situation and based on indicators is above average. Based on the results of the research, it was found that the organization distributes knowledge in order to make it publicly available, encourages managers to disseminate knowledge to each other, disseminates knowledge through seminars, conferences, workshops and also disseminates knowledge through regulations and Organizational guidelines have been relatively successful, with good results during exposure and management of Covid 19 disease.

Also, based on the obtained results, it was found that the organization has been successful in transferring knowledge with the aim of public access, encouraging managers to transfer knowledge to each other, transferring knowledge from regulations, rules and face-to-face meetings and lectures. The results of the study also show a better performance in terms of exposure and management of Covid 19 disease in the field of knowledge transfer and dissemination compared to other studies. In a similar study, knowledge distribution in Isfahan University of Medical Sciences was below average [13]. Also, the general situation of the military research center in terms of knowledge-oriented and components of knowledge transfer and dissemination is below average (between average and weak). However, in the present study, 
managers rated the status of knowledge transfer and dissemination at the time of exposure and management of Covid 19 disease as above average [14]. Knowledge management in the emergency room of Hazrat Rasool Akram Hospital was also classified among the eight categories of Baldrige excellence model in the third level of excellence that the scores obtained in the field of dissemination and transfer of knowledge show less than the present study [15]. In the study of knowledge management status among the staff of Tarbiat Modares University, it was found that 3.8\% of the knowledge management staff rated the status of its components as strong, $76 \%$ as moderate and $26 \%$ as weak. [16]. Also, knowledge dissemination and distribution in Isfahan Steel Company was determined with 95\% confidence below the average level. In this research, better results were obtained in terms of knowledge dissemination component, which shows that knowledge dissemination has a favorable status in the research organization [17].

\section{Conclusion}

According to the results of the present study, it can be seen that considering the competitive advantages in today's world, organizations that are active in the field of knowledge management and have taken good steps, in order to apply knowledge, it is necessary to prepare and implement appropriate planning for the transfer and dissemination of knowledge with the participation of users (managers and employees). What is clear is that government organizations are basically bureaucratic and that the transfer and dissemination of knowledge in them is associated with difficulties and people are less willing to share the knowledge they have with others so that the organizations gain the knowledge of power and can improve their situation. In the present study, which was conducted at the headquarters of the Ministry of Health and Medical Education as a government complex, I t was found that in times of crisis and special conditions such as exposure and management of Covid 19 disease greatly facilitates the transfer and dissemination of in government organizations and its managers, which is confirmed by the high scores in this regard. Undoubtedly, one of the strategies to apply knowledge management in educational, medical, support and research units and health and medical organization, especially in the public sector and in times of crisis, is the dissemination and transfer of knowledge. Therefore, establishing a source or manual and digital repository of documents and equipping it with all upto-date information and data related to Covid 19 disease management and encouraging managers to use its data and information are the most important suggestions of the present study in order to improve the conditions for dissemination and transfer of knowledge, the path of exposure and management of Covid 19 disease in the headquarters of the Ministry of Health and Medical Education.

Also make available data and information in the organization, especially raw and processed data for the use of managers related to Covid 19 disease management, encourage staff to share their knowledge of Covid 19 exposure and management in the organization, Creating a system for attracting and using people with knowledge, using past experiences to solve existing problems ,using stored information and knowledge, creating an incentive system for exchanging and disseminating knowledge, designing appropriate databases for Covid19 disease exposure and management and trying to turn raw data into information, then into knowledge and finally wisdom in the organization is one of the main suggestions of the present study. It is also suggested to encourage employees to disseminate knowledge in the form of workshops, seminars, Lectures and the like should be done in webinars and online. It is also suggested that other components of knowledge management at the time of exposure and management of Covid 19 disease and other environments dealing with Covid 19 disease, such as medical universities, hospitals and health networks be studied so that the status of knowledge management in the entire health care system of the country at the time of exposure and management of Covid 19 disease is considered.

\section{Compliance with ethical standards}

\section{Disclosure of conflict of interest}

No conflict of interest.

\section{Statement of informed consent}

Informed consent was obtained from all individual participants included in the study.

\section{References}

[1] Ho LA. "What affects organizational performance? The linking of learning and knowledge Management", Industrial Management \& Data Systems. 2008; 108(9) :1234-1254. 
[2] Jasimuddin SM. "A holistic view of knowledge management Strategy", Journal of Knowledge Management. 2008; 12(2): 57-66.

[3] Carniro A. The role of Intelligent Resource in know ledge Management. Journal of K.M. 2001; 5(4): 43-55.

[4] Proust, Gilbert, Robb, Stephen and Mahartet, Kai Ro. "Knowledge Management", translated by Ali Hosseinikhah, Citron Publications, Tehran. 2006.

[5] Bose R. Knowledge management-enabled health care management system ‘ capabilities, infrastructure, and decision-support. Expert Systems with Applications. 2003; 24: 59-71.

[6] Beveren JV. "Does health care for knowledge management? Journal of Knowledge management. 2003; 7(1): 9095.

[7] Shahgholian, Keyvan. "Designing a model for evaluating the level of knowledge management in industrial organizations in Iran", PhD thesis in Industrial Management, Islamic Azad University, Science and Research Branch, Tehran. 2005.

[8] El Miller, William and Morris, Lancron, "The Fourth Generation of R\&D in Knowledge Management, Technology and Innovation", translated by Alireza Mohajeri and Maryam Fattahzadeh, Jihad Daneshgahi Publishing Organization, Tehran. 2004.

[9] Management and Planning Organization, "Law of the Fourth Economic, Social and Cultural Development Plan of the Islamic Republic of Iran (2005-2009)", Deputy of Administrative, Financial and Human Resources Affairs of the Documentation Center, Tehran. 2004.

[10] Narteh B. "developed-developing country interfirm collaborations ، a conceptual framework", Journal of Knowledge Management. 2008; 12(1): 78-91.

[11] Rhodes J, Hung R, Lok P, Ya-Hui Lien B, Wu C. "Factors influencing organizational knowledge transfer ، implication for corporate performance", Journal of Knowledge Management. 2008; 12(3): 84-100.

[12] Sedighi Jila et al. "Designing a" knowledge translation "model to benefit from research results", Payesh. 2007; 7 (1): 35-47.

[13] Shirvani Alireza, Safdarian Ali, Alavi Azadeh, "The Rule of Knowledge Management Processes in Isfahan University of Medical Sciences", Health Management. 2009; 6 (1): 75-82.

[14] Rafati Shaldehi, Hassan, Hasnavi, Reza, Beh Azin, Farid, Bani Taba, Seyed Alireza, "Study of knowledge management model in a military research center", Military Medicine. 2008; 10 (3): 237-252.

[15] Maleki Mohammad Reza, Nasiripour Amir Ashkan, Aghababa Sara, "Knowledge Management in the Emergency Room of Hazrat Rasool Akram Hospital (PBUH) Based on Baldrige Excellence Model: 2007", Health Information Management. 2007; 4 (2): 167-174. 